(C) Springer-Verlag Berlin Heidelberg 2007. Access to this work was provided by the University of Maryland, Baltimore County (UMBC) ScholarWorks@UMBC digital repository on the Maryland Shared Open Access (MD-SOAR) platform.

Please provide feedback

Please support the ScholarWorks@UMBC repository by emailing scholarworks-group@umbc.edu and telling us what having access to this work means to you and why it's important to you. Thank you. 


\title{
A Non-Visual Approach To Improving Collaboration Between Blind And Sighted Internet Users
}

\author{
Ravi Kuber, Wai Yu \& Graham McAllister \\ Sonic Arts Research Centre \& Virtual Engineering Centre \\ Queen's University Belfast \\ Belfast, BT7 1NN, UK \\ \{r.kuber, w.yu, g.mcallister\}@qub.ac.uk
}

\begin{abstract}
Assistive technologies have widened access to the Web, by providing a non-visual representation of page content for blind individuals. However, restrictions imposed by screen readers and Braille output devices have led to the development of mental representations of a web page, which differ considerably from the visual layout. As a result, both blind and sighted users find it difficult to perform joint web-based tasks. In this paper, we have applied a structured participatory-based approach to develop haptic sensations to assist the collaborative process. Preliminary results are reported and on-going development is discussed. It is anticipated that findings from the study will inform a haptic framework, which will assist developers when designing an accessible collaborative interface, using the benefits which the sense of touch can offer.
\end{abstract}

Keywords. Blind, collaboration, design methodology, haptic, Web accessibility.

\section{Introduction}

There are over two million people living with sight loss in the UK [9], many of whom use the Web for purposes of work, education and leisure. Screen readers and refreshable Braille displays are commonly used by individuals unable to rely on their functional vision, to access the contents of a page. However, these assistive technologies are known to impose restrictions on the browsing process, forcing blind users to move sequentially through each page. Items such as graphics and animations are inappropriately handled, and structural information is often omitted. As a result, developing an overview of a page can pose a challenge [14]. While previous studies have mainly focused upon assisting blind users when independently exploring a web page, research has indicated that additional support is required to collaborate on web-based tasks with sighted peers [7]. Examples include working with colleagues to design web sites, and with telephone-based advisors to complete a series of web-based forms or to download and install software from a page [4,7].

Blind participants often find the process of receiving directions from sighted users challenging, as it is difficult to locate or reach a particular target on an interface, using commands provided by an assistive device. As few screen readers and Braille output displays offer a visual marker, sighted users are often left confused as to a blind user's position on a web page. This can lead to dependence on the sighted user to complete the task. To facilitate joint operation, visual and non-visual interfaces should ideally support the same model of the application interface, and their respective presentation should be synchronised [8]. However, the lack of positional information provided by assistive devices, can lead blind users to envisage the layout of a web page as a vertical column-like structure, rather than the rich visually-dense representation perceived through sight $[7,8]$. Developing a sense of coherence between both visual and non-visual web interfaces would enable both groups to work together towards a common goal.

\section{Related Work}

Research has shown that non-visual technologies are beneficial to the performing a collaborative task $[1,10,11,12,13]$. Winberg and Bowers [13] have presented sighted users with a graphical interface and blind users with a corresponding auditory interface. Participants were asked to play the Towers of Hanoi game, moving discs from one tower to another. Findings showed that blind participants were able interact with the sonified environment, taking turns to move discs. They were also able to monitor 
the moves made by their sighted partners, leading to completion of the task. Sanchez et al. [10] performed a similar study presenting blind participants with an auditory battleship game with a tactile grid. Blind participants were able to mentally map the non-visual interface. The experience for both blind and sighted children was thought to be both entertaining and challenging.

The sense of touch is also known to be a powerful modality for interface design, providing vital spatial and navigational cues needed to access a non-visual environment [6]. Crossan and Brewster [1] have discussed the design of a collaborative maze game for visually impaired and sighted children using the benefits offered by haptic technologies. The researchers have discussed the benefits presented by the introduction of a secondary device to aid awareness of position, promoting interaction with the virtual environment. Salnäss et al. [11] have asked visually impaired and sighted participants to locate, identify and manipulate objects using a haptic device. Results showed that visually impaired participants were able to form a mental representation to support orientation, and navigate through the environment guided using deictic references (e.g. "move left, right, up etc") from sighted users.

It is thought that non-visual technologies can be used to assist blind and sighted users when performing a web-based task. Developing haptic sensations which can be accessed using traditional graphically-oriented browsing applications (e.g. Internet Explorer or Firefox) would enable blind users to gain the assistive feedback necessary to explore a web interface. At the same time, sighted users would be able to browse the Web in the manner which they are accustomed to.

\section{Architecture}

An assistive tool has been developed by our research group [14] which enables blind people to explore a web interface using the benefits offered by multimodal technologies. A force-feedback mouse is used for navigating around the interface, and its cursor position is constantly monitored by a contentaware plug-in. As the user moves within close proximity to an element located on a web page, appropriate haptic and auditory (speech and non-speech) prompts can be presented [14]. Assistive guidance towards a particular target can also be provided.

Findings from a study have revealed that the multimodal interface provides a usable alternative to a screen reader for purposes of exploring the Web. Haptic technologies were found to be influential to enabling participants to locate interface objects (e.g. images and hyperlinks) and develop a spatial representation of page layout [14]. However, the evaluation focused on independent exploration of the Web, rather than how participants would manage to work together on web-based tasks. Research has indicated that if an accessible user interface is developed, there is no guarantee that collaboration will necessarily be supported $[12,13]$.

\section{Approach}

In order to design assistive haptic sensations which can support blind individuals when performing a joint task with sighted users, a structured method should be adopted. A five-step approach proposed and detailed in an earlier publication [3], has been used to assist blind users when exploring the Web independently. The method has since been modified, and applied to the context of collaboration (Fig. 1).

- Step 1 - To observe blind and sighted users working together, a series of web-based tasks are designed and presented to pairs of blind and sighted participants. Blind users are asked to use their screen readers to perform the tasks, and encouraged to "think aloud", verbalizing intended goals and outcomes of any actions made. The session is video recorded, and a hierarchical task analysis is performed on the footage. The aim of the step is to analyse the textual or diagrammatic representations from the task analysis, to assess the challenges faced when using a screen reader, and to examine areas where additional assistance would be beneficial.

- Step 2 - Another series of web-based tasks are designed and presented to pairs of blind and sighted participants. Blind users are observed whilst interacting with a multimodal interface and accompanying haptic device to complete the tasks set. They are asked to discuss their thoughts regarding haptic feedback present on the interface. The session is video recorded and a hierarchical task analysis is also conducted. The aim of this step is to assess whether the haptic sensations provide enough support for the collaborative process, and to consider ways in which further assistance can be provided to support joint tasks between blind and sighted users. 
- Step 3 - On the basis of the findings from the analyses, a scenario relating to the haptic exploration of a web interface can be constructed. If findings from earlier observations have revealed that considerable time and mental effort is spent trying to perform a task with the screen reader or with the haptic device, this finding should be included in the scenario for purposes of discussion. Prototypes should be developed by the researcher, to adhere to the needs of the users when performing a collaborative task.

- Step 4 - Assemble a design team (also referred to as user group) consisting of haptic interface designers and blind screen reader users. The researcher should lead the group through the main issues covered by the scenario. The aim of this step is for the group to verify whether the prototypes serve their purpose, and provide further feedback to strengthen them. Alternatively, the group can suggest other design ideas. To do this, participants should think about the role that haptic feedback would play on the interface, and then consider special qualities that touch has to offer. Sensations should not be considered in isolation. If participants make a design suggestion to aid collaborative work, they should consider whether the sensation would work in conjunction with mappings defined for use on the web-based interface. These suggestions can be developed and presented to successive design teams.

- Step 5 - Assemble further user groups comprising of blind screen reader users and haptic designers, and present them with the same scenario, and prototypes resulting from steps 3 and 4 . This stage aims to iterate designs until they reach an acceptable standard. At this point, representations can be placed within the assistive haptic framework.

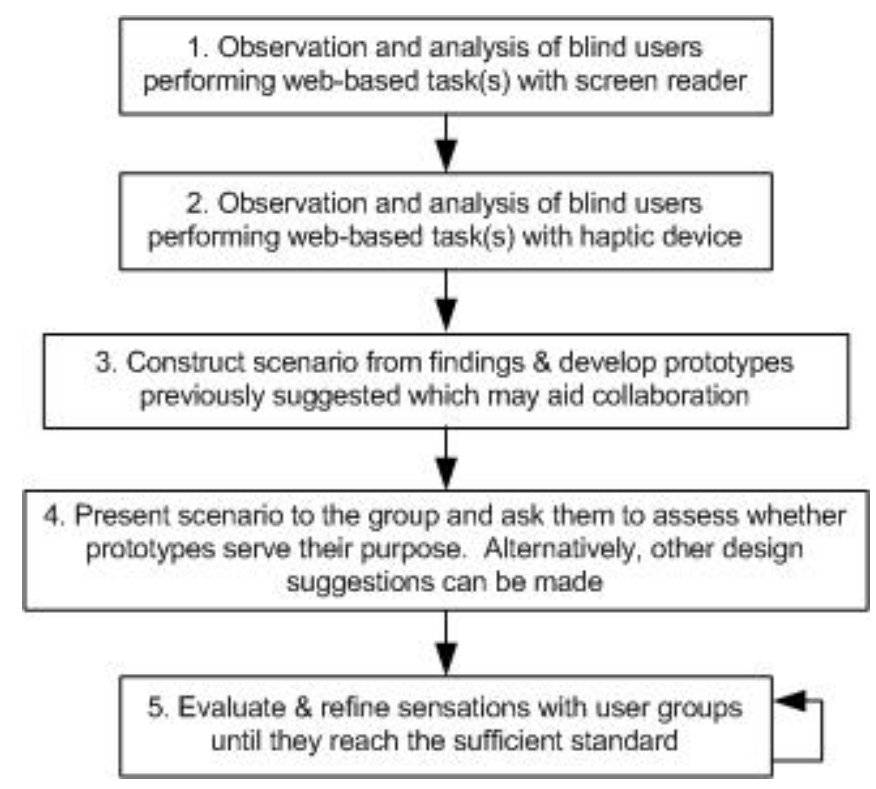

Fig. 1. Five-step approach applied to develop haptic sensations for the purpose of collaboration

\subsection{Step 1: Observation and Analysis of Blind Users Performing Tasks with Screen Reader}

Six blind volunteers, aged between 18 and 32, were recruited for purposes of the study. Their levels of sight ranged from limited functional vision to complete occlusion. All the volunteers used a screen reader to access the Web.

Each participant was paired with a sighted user (played by the evaluator), and presented with a task. The objective was to move through a series of five unfamiliar web pages, to find a piece of information present on the final page. To complete the task, blind participants were required to locate a particular target (e.g. a hyperlink) present on each web page, using a screen reader. Directions to locate the target were provided by the sighted participant. If the appropriate target was identified and selected, a new web page would open, where a new target would be located. Blind users could ask their sighted partners for additional directions. However, the onus was on the blind users to navigate towards each target using keyboard shortcut commands. No time limits were imposed to complete the task.

The first task presented was to locate a hyperlink entitled 'Research Project' on the web page (Fig. 2). Participants used a variety of strategies to locate a hyperlink, with some using shortcut commands (Insert + F7 keys) to display a list of hyperlinks present on the page. Others attempted to locate the 
Pre-print: Kuber, R., Yu, W. \& McAllister, G. 2007: A Non-Visual Approach to Improving Collaboration between Blind and Sighted Internet Users. In proceedings of Human Computer Interaction International'07 (Lecture Notes in Computer Science 4556), 913-922.

hyperlink by moving sequentially through the hyperlinks present on the page. Most participants tended to pause and select a similarly entitled hyperlink 'Project' (Fig. 2). However, this was not the intended target. If participants would have continued moving through the page or list of hyperlinks, they would have eventually located the actual target 'Research Project'. A clearer overview of contents of the page could provide more awareness of the range of elements present on a web page. This could reduce the number of errors made targeting incorrect items.

Participants asked to locate a hyperlink in the second paragraph, roughly positioned in the middle of a page (Fig. 3). None of the participants knew the specific screen reader commands for navigating to a particular paragraph or to the central region of the page. Furthermore, one participant asked the sighted evaluator to explain how the paragraph would appear visually, and how it would differ from a regular text present on a page. Similar comments were made about the distinction between the appearance of textual hyperlinks and image-hyperlinks, as many participants did not realise that there was a difference between the two. The reason for this is because screen readers often use similar terms to identify each object ('link' and 'link-graphic'). It seemed evident that the terminology used by sighted users both in to describe items on a page, and for purposes of giving directions, is different to that used by blind users.

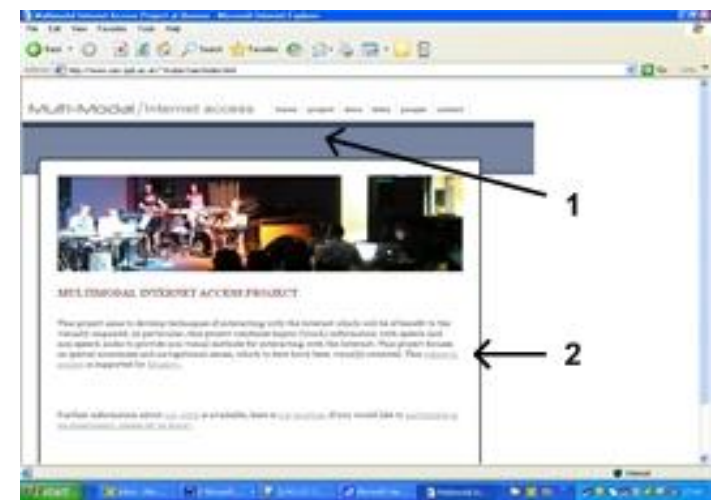

Fig. 2. Participants often paused at (1), instead of moving through the page to locate the actual target (2)

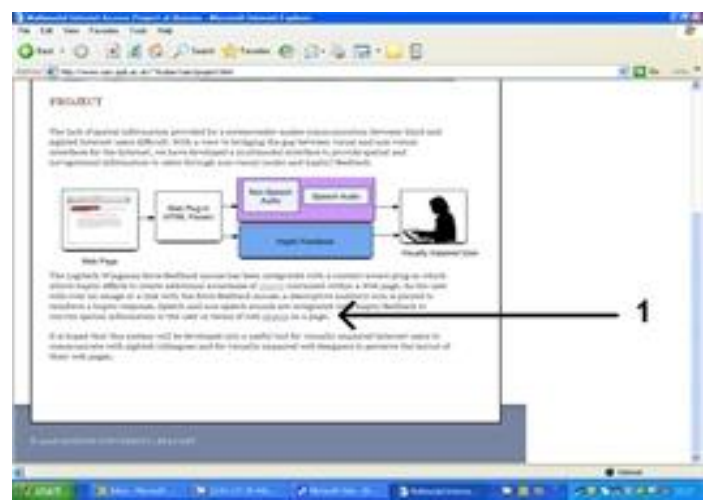

Fig. 3. Some participants found it difficult to locate a hyperlink interspersed within a paragraph of text (1).

Participants were informed that target to search for, was interspersed within a paragraph of text (Fig. 3). As the hyperlink label formed part of the sentence, they would need to 'read' the whole sentence to understand the meaning associated with the hyperlink target. Some of the less experienced users were unsure of how to navigate through text with hyperlinks embedded within it, as they had never encountered a situation like this previously. Participants devoted time and mental effort, using a combination of arrow key and page down commands to locate the information to check and doublecheck that they had reached the correct hyperlink.

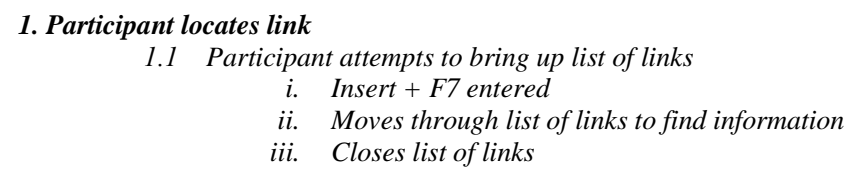

1.2 Participant moves through links using traditional method

$i$. Listens intently for keyword LINK and keyword AUDITORY INTERFACE using auditory feedback

Fig.4 Textual representation of tasks performed by one blind user attempting to locate the target associated with a particular publication.

Participants were then asked to locate a PDF document associated with a particular publication listed on the web page. The majority of the participants used a command to display a list of hyperlinks, and began searching through the list to identify the target. However, all the hyperlinks present on the page, were labelled using the term 'Download PDF'. The participants who used the Tab key to move through the sequence did not realise that the order of elements differed to the presentation of links on the page. This meant that had the participants been working with a sighted user, who asked them to locate the third link present on the page, the blind user would have selected the wrong document for download. Participants stated they commonly encountered the problem of poorly labelled or 
Pre-print: Kuber, R., Yu, W. \& McAllister, G. 2007: A Non-Visual Approach to Improving Collaboration between Blind and Sighted Internet Users. In proceedings of Human Computer Interaction International'07 (Lecture Notes in Computer Science 4556), 913-922.

inappropriately ordered information. They found it easier to use the Arrow keys to move through each element present on the page, listening very carefully to the accompanying publication details associated with each hyperlink (Fig. 4). Concentration and time was needed for this task. Additional awareness of element positioning would have assisted the users in this case.

\subsection{Step 2: Observations and Analysis of Blind Users Performing Tasks with Haptic Device}

Haptic sensations were developed using an earlier application of the five-step methodology [4], to represent images, hyperlinks, image-hyperlinks and borders (Table 1) ${ }^{1}$. These mappings were then integrated into the assistive tool described by Yu et al. [14].

Table 1. Haptic mappings used on web interface

\begin{tabular}{|l|l|}
\hline \multicolumn{1}{|c|}{ Elements } & \multicolumn{1}{c|}{ Haptic Mappings } \\
\hline Images & $\begin{array}{l}\text { A slightly raised enclosure effect is used to encase the visual border of the image. A weak } \\
\text { spatial texture is applied to the image's interior. }\end{array}$ \\
\hline $\begin{array}{l}\text { Image- } \\
\text { hyperlinks }\end{array}$ & $\begin{array}{l}\text { A multi-dimensional spring effect is used to direct the user towards the relative centre of an } \\
\text { image-hyperlink. A spatial texture effect (the same texture applied to images) is applied to } \\
\text { provide awareness of the element. }\end{array}$ \\
\hline Hyperlinks & $\begin{array}{l}\text { A multi-dimensional spring effect is used to direct the user towards the relative centre of a } \\
\text { hyperlink. A weak periodic wave effect to alert presence of the hyperlink body. }\end{array}$ \\
\hline Page border & $\begin{array}{l}\text { A lowered enclosure effect is used to provide a border around the HTML content. This } \\
\text { allows the user to explore the page, and perceive its boundaries. To leave the page, the user } \\
\text { is able to push-out on to the raised border. A vibration is encased around the border, to } \\
\text { provide additional positional awareness for the user. }\end{array}$ \\
\hline $\begin{array}{l}\text { Page } \\
\text { background }\end{array}$ & $\begin{array}{l}\text { No haptic sensation should be used to represent the background, as the other mappings on } \\
\text { the page should stand out. The location of text on a web page should not be communicated } \\
\text { through haptic technologies. }\end{array}$ \\
\hline
\end{tabular}

Fourteen blind volunteers, aged between 18 and 65, were recruited for the study. All participants used a screen reader to access computers, as they experienced limited levels of functional vision or complete occlusion.

The aim of the task was to investigate whether both blind and sighted users could work together towards a common goal. The scenario of working with a telephone-based advisor to perform an installation of a web-based plug-in was presented. Each partnership would be required to move through a series of six unfamiliar web pages, to locate and install the plug-in. Each blind participant was asked to use the force-feedback mouse to explore the assistive interface, and locate certain targets (e.g. hyperlinks, image-hyperlinks) present within each page. Directions to each target were communicated verbally by the sighted user (played by the evaluator). Each selected target would open a new page, where another target would be located. This process would continue until the final target on the sixth page had been located, signalling that the plug-in had successfully been installed. The sighted user was unable to view movements made by the blind users, relying on their verbal feedback to communicate their current location on the page. A time limit of ten minutes was imposed to explore each page. An extended version of the procedure is presented in Kuber et al. [4].

Findings from the study revealed that blind participants were able to perceive and identify objects presented on the web interface. They were able to locate a particular landmark on a web page (e.g. large image or hyperlink), and use this as a basis of communicating their spatial position to the sighted user. In turn, the sighted user would provide directions to another fixed reference point within the page. This process would continue until the blind user had located the actual target (average 61.09 seconds). All participants managed complete the series of steps needed to install the web-based plugin. By exploring the page, participants could use the spatial feedback presented to construct a mental map of object layout. Through the course of the experiment, blind participants' levels of confidence appeared to grow using the haptic device to follow spatial directions. It seemed that blind and sighted participants had developed the shared mental understanding of interface layout, discussed by Salnäss et al. [11].

Many participants adopted interesting strategies to support exploration on the interface. Borders were used by some participants as a 'ruler', providing a means to move in a straight direction to

${ }^{1}$ Since the experiment, a further session was conducted to iterate findings, discussed by Kuber et al. [3] 
another section of the page. Other participants would use the border as a fixed reference point, to try and ascertain their relative position on the interface, by gauging the distance to the nearest corner. This technique did not always provide an effective representation as participants noted that making small movements with the mouse would often correspond to larger movements on the interface.

Participants seemed to be able to locate and target larger hyperlink targets quite effectively. The attractive feedback was found to be helpful, as it reduced the opportunity to deviate from the central region of the hyperlink. As none of the blind participants had previously used a mouse, they were unfamiliar with the concept of clicking the left mouse button while still holding the mouse. Many would locate a hyperlink using the haptic feedback presented on an interface, and then move their hand away from the body of the mouse, to click the left button. As a result, the mouse pointer would move from the hyperlink, and the target would need to be reselected. The majority of participants appreciated the use of a weak vibration which helped provide awareness of link position on the interface. They were able to use this sensation to align the mouse over the link to select it. Other participants suggested that vibrations may become too overloading on pages which contain a number of hyperlinks, such as web portals. A less intrusive method of providing awareness could provide benefit.

Areas for Improvement. Analysis revealed that participants experienced the following issues relating to spatial orientation and navigation on a web interface:

- Assessing current position on the page - Participants had no quick method of assessing their current spatial position on the interface (e.g. whether they were at the left or the right-hand side of the page), and communicating this information to a sighted user. Some participants were forced to spend time attempting to isolate an object (landmark) to try and convey current location.

- Moving along a straight path - Participants stated that they could navigate in the direction specified by the evaluator when using the force-feedback mouse. However, they were unsure as to whether they were adopting a straight path or deviating in another direction altogether.

- Locating elements on the interface - In some instances, certain elements proved difficult to locate on the interface. Problems mainly occurred when attempting to find small targets (e.g. textual hyperlinks and image-hyperlinks) within large empty regions of a page, due to the lack of surrounding feedback. Time and mental effort would be required to explore the interface to isolate these targets.

It is thought that haptic sensations could be developed to support the three areas identified.

\subsection{Steps 3 \& 4: Developing and Presenting Scenarios and Prototypes to Design Team}

From the results of steps $1 \& 2$, a scenario was constructed highlighting the needs of Alison, a blind user with no functional vision, performing a task in conjunction with her sighted colleague, Laura. As Laura sits at a desk facing Alison, she is unable to view actions made by Alison when exploring the Web. The task selected was to access a time management system to enter in weekly hours. It was chosen because one blind participant had explicitly mentioned in an interview, her problems using a screen reader to enter data into a web-based form. As a result, she would ask sighted users for assistance. However, due to the limited coherence between visual and non-visual presentation, she would often ask sighted participants to complete the task on her behalf [4]. Prototypes to assist navigation and orientation were developed by embedding haptic sensations within static HTML pages. These were based on design ideas provided by blind screen reader users and haptic designers in an earlier study [3]. An excerpt from the scenario is shown below:

Laura asks Alison to open-up the time management web page, and to navigate towards a large image. Alison explores the page, but cannot seem to locate any elements other than plain text. Laura asks Alison about her current location on the page. Confused, Alison makes a keystroke, turning on an optional haptic effect allowing her to identify her position. Using the haptic sensation provided, she is able to ascertain that she is located at the left-side of the page. This allows Laura to redirect Alison towards the button located towards the bottom of the page, to open the web-based form.

The scenario was presented to the design team comprising of one blind haptic interface designer, one sighted haptic interface designer and one blind auditory interface designer. The design team would be able to listen to the scenario, interact with prototypes, and suggest whether sensations designed could target the three issues identified. They could then discuss amongst themselves how to improve 
the haptic feedback, or alternatively suggest new ideas for design. It was hoped that any findings would not be solely constrained to filling out web-based forms for time-management systems, but also apply to other collaborative contexts of use.

Assessing Current Position on a Page. Findings from Kuber et al. [3] had revealed that to assess the current position on a web page, participants had suggested providing two distinctive sensations, one at the either side of the page. Both effects should merge into one another towards the centre of the interface. It was thought that if the user was unable to locate a distinctive landmark to communicate his/her relative position on the interface, he/she could use the optional sensation to provide awareness of location. For purposes of testing, participants were asked to explore an interface with a spatial texture at the left side of the page, and a temporal vibration at the right, as these effects were thought to be the most distinctive from one another. Results showed that all members of the design team were able to differentiate between the texture and vibrations. They were able to detect that each of the sensations varied in strength depending on the user's relative location on the page (i.e. sensations were stronger towards the ends of the page, and weaker towards the centre).

Participants were presented with a web page containing a number of images and the haptic orientation effect designed (Fig. 5). They were asked to identify the position of each image on the interface, and count the number of images present. Results showed that both blind users were able to locate all the images on the interface, despite the presence of additional feedback on the background. They were able to accurately identify the locations of each image on the page. However, the effect was thought by participants to be inappropriate for purposes of detection, as it was a time consuming method to identify object location. This was due to the amount of haptic feedback present on the page. One participant stated that the application of the effect on the interface would result in confusion, and not add any value to the interface. It was thought that more value could be gained from using the border as a means for referencing location. Discussion between members of the group inspired the blind haptic designer to suggest an idea for improving the effect. It was thought that four different haptic effects could be placed along the page border, one to mark the position of each quadrant (Fig. 6). If the user wished to identify location on the page, he/she could move to the border and gain positional feedback in a less intrusive manner. This sensation would be mocked-up and tested with a new user group. The use of notches embedded within the border was briefly discussed by the group, as a method for locating position within the page. However, the idea was later rejected, as they thought that counting notches on the border would involve too much time being spent.

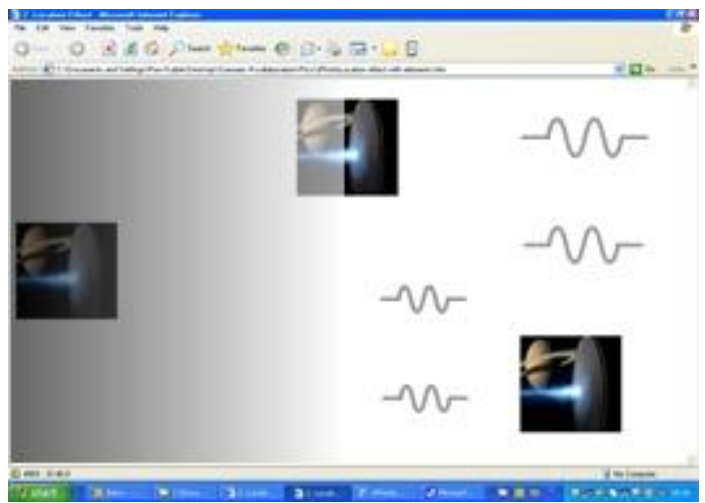

Fig. 5. A strong spatial texture present on the left-hand side of the page, reduces in intensity as the user moves rightwards. Similarly, a strong temporal vibration at the right-hand side of the page weakens as the user moves leftwards.

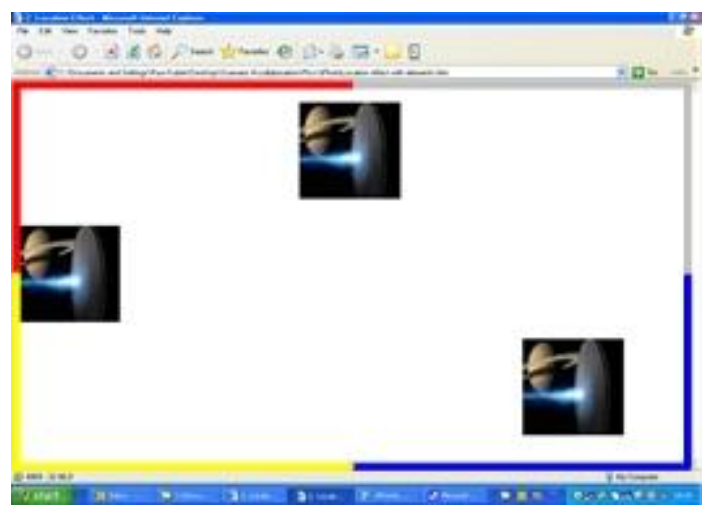

Fig. 6. No additional feedback is applied to the interior of the page. However, the border effect is supplemented with additional haptic sensations, differentiating each quadrant.

Moving along a Straight Path. Participants had previously discussed the use of tunnels or grooves, as a method of moving in a line without deviating from the path [3]. Haptic tunnels were developed using the findings from Langdon et al. [5], where spring effects are embedded in each wall, separated by a channel width of 20 pixels. As the cursor passes over a tunnel wall, a spring force pulls the cursor to the inside wall [5], providing awareness of the path to be followed (Fig. 7).

As participants began to navigate through the tunnel, they tended to move from side-to-side hitting the inner walls, until they became more familiar with the path. All members of the design team 
thought the effect was appropriate for use, providing a fairly non-intrusive method of moving along in a straight direction. The blind haptic designer stated that she could feel enough resistance against the walls, enabling her to identify the limits of the channel. When asked how the effect could be improved, suggestions were made by both haptic designers to reduce the size of the springs, and improve the offset values to ensure that the user could take a clearer path through the channel. The blind audio designer thought that this improved effect would also be useful for guiding beginners (like the character from the scenario) from a search box to a submit button, or to move to areas of interest, aiding independent exploration, in addition to collaborative work. Participants felt that the tunnel would be better than other attractive effects discussed, as this effect allowed them to be in control of movement, rather than being propelled to a particular target located in another region of the page.

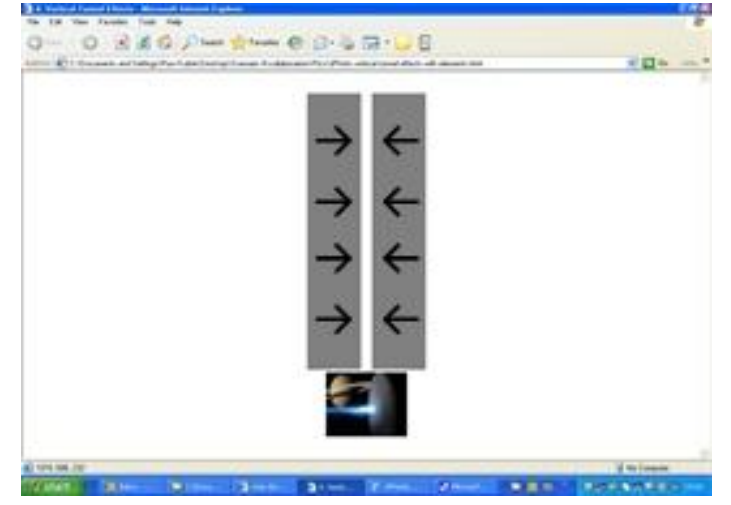

Fig. 7. Vertical haptic tunnel created with 20 pixel channel width. As user moves downwards through the channel, he/she is able to locate the element positioned that the bottom of the page.

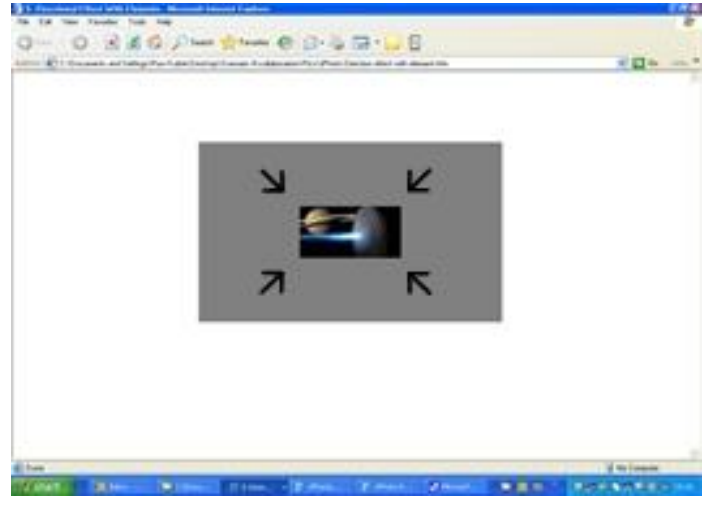

Fig. 8. Periodic wave increases as user moves towards small target positioned within the page. Alternatively, user can be guided using attractive motion.

Locating Elements on the Interface. Findings from Kuber et al. [3] had revealed that periodic waves increasing in intensity when approaching an object, would provide additional guidance to a user. Gentle effects attracting the user to an element located in close proximity had also been discussed. In the current study, participants were introduced to two effects (Fig. 8); periodic wave increasing in intensity and constant motion effect designed to move the mouse to the intended location. The effects surrounded small images, rather like a haptic aura. Both effects were designed to provide a subtle form of directional assistance.

Participants generally found both sensations quite useful to provide guidance to the intended targets. Both blind participants in the group found the directed motion more effective than the periodic wave effect. This was because directed motion would physically guide the user to target the area of interest. However, the sighted haptic designer felt that there was more information present within the periodic wave increasing in strength enabling the user to determine the relative proximity towards the element. The other members of the group suggested that periodic sensations would conflict with other periodic wave mappings (Kuber et al., [3]). To gain consensus on the most appropriate effect, both would need to be tested with successive user groups, to ascertain the respective benefits and weaknesses for purposes of locating a small element on an interface.

\subsection{Step 5: Evaluating and Refining Sensations}

From the basis of findings, prototypes will be improved and implemented in the multimodal plug-in. Further sessions will be conducted with design teams, to assess whether the sensations suggested provide benefit to blind users when collaborating with sighted peers. In order to ensure that sensations are refined to a sufficient standard, designs can be evaluated against the same scenario, and against additional scenarios exploring other contexts of use (e.g. jointly booking a ticket or installing a webbased plug-in). More comprehensive testing over a longer period of time will reveal how effectively feedback can be used to aid the collaboration process. Sensations would then be placed in the assistive framework accordingly. 


\section{General Discussion}

The five-step approach has been used to isolate some of the problems faced by blind screen reader users, when attempting to work on web-based tasks with sighted users. It seems apparent that blind and sighted users develop different mental representations of their respective interfaces, which contribute to problems with communication and collaboration. Haptic technologies have been shown to be vital to aiding blind users to access interface objects and visualise layout of a page. Blind users were able to locate landmarks which could be used to communicate relative position to sighted users. Both parties were also able to communicate in terms of directions, which could not always be achieved through use of a screen reader. As a result, blind and sighted users were able to work together to achieve a common goal.

Winberg [12] has stated that the non-visual presentation of the interface should be sufficient to let the blind user play an active role in the collaborative process and help to solve problems. Even though findings have showed that blind and sighted users were able to develop a shared mental representation of the interface, further observation and analysis of blind users working with a haptic device revealed that additional navigational and orientation support would be required for purposes of collaboration. As a mouse offers a predominantly visual-centric approach to exploration, blind users prefer to make use of keyboard shortcut commands in conjunction with screen readers and refreshable Braille displays. However, when a blind user interacts with a device such as a mouse, additional assistance is needed to make the precise movements required, and monitor the relative cursor location on a web interface as unintentional and unnoticed rotations can be made [2]. The results of our experiments confirm the benefits of locational effects (auras) and haptic tunnels to assist the navigation process. Gentle attractive forces have been shown to be useful to guiding users to a small target. However, forces should be subtle enough to allow the user to resist it, while at the same time strong enough to attract the user to the target once the grip is reached [6]. In terms of the haptic tunnel used, Langdon et al. [5] thought that the freedom allowed by the presence of the wide channel could possibly be a hindrance to motion impaired users experiencing difficulty navigating in a straight line. However, the width of 20 pixels was thought to be adequate for blind users new to a mouse, as it provided them with the directional information necessary to follow the channel and complete the task. Participants did not feel constrained by this effect. The testing of the haptic effect to assess current position on the interface, has revealed that the overuse of haptic feedback on the interface can be counter-productive, and may reduce the quality of the subjective browsing experience. Sensations should be usable and should support the user in his/her intended task.

Individual differences should also be taken into account when using haptic sensations to provide a non-visual representation of a web page. Certain users may require stronger feedback than others, so a setting rather like the 'volume dial' of a radio was suggested for tailoring effects to user preferences. Magnusson et al. [6] have also discussed the importance of tailoring the strength of forces to the type of hardware used.

The importance of context has been emphasised by Winberg [12] when studying collaboration between blind and sighted users. Issues such as location, motivation and the experiences of people should be taken into account when the task is being designed. In the study, a real world situation of contacting a helpdesk advisor was recreated, and findings were observed. The scenario provided a useful means to evaluate prototypes and solicit design ideas for assistive feedback. The user groups were generally able to identify with the visually impaired character in the scenario interacting with haptic feedback, and were able to provide suggestions even if they had limited experience of a screen reader or haptic technologies. The participatory-style approach enabled both haptic interface designers and blind users to discuss and reflect upon each other's ideas, bringing the strengths of their own knowledge and experiences to the design table. In the participatory-design process, it is important to remember that end users are not expert designers. Users may make design suggestions, but these may not necessarily be appropriate or feasible for use on an interface. Testing should take place to ensure that suggestions fit their purpose, and work in conjunction with other mappings.

While haptic feedback is thought to serve as a substitute for the visual mode [11], it has been acknowledged that there are restrictions on the information that haptic modality can provide. The haptic modality is limited in bandwidth, and devices are one-point in nature, limiting the extraction of attributes. Haptic feedback can be used to provide the majority of spatial and navigational information on an interface. However, it is not always appropriate to represent certain features (e.g. to quickly assess current position on a page), which may be better conveyed through another modality such as speech or non-speech audio. 
Pre-print: Kuber, R., Yu, W. \& McAllister, G. 2007: A Non-Visual Approach to Improving Collaboration between Blind and Sighted Internet Users. In proceedings of Human Computer Interaction International'07 (Lecture Notes in Computer Science 4556), 913-922.

\section{Conclusions and Further Work}

This paper has applied a structured approach to develop assistive haptic feedback to the context of a collaborative web-based task. Results have shown that haptic sensations are able to provide the spatial and navigational support needed. Application of the method has shown that design ideas can be developed and tested, targeted to the needs of blind Web users. Discussion can be generated amongst haptic interface developers and blind screen reader users to suggest new ideas which can be evaluated accordingly. As a next step in the research project, it appears pertinent to refine and test mappings. Further experiments, observing blind and sighted users performing the tasks in a more natural environment would serve as a better indicator of assessing the merits and weaknesses of haptic feedback on a page.

Acknowledgements. We wish to thank the volunteers who participated in the study. We are also grateful to Emma Murphy, Dr Sile O’Modhrain, Philip Strain and Samantha Chui-Chui Tan for their help and advice. This work is supported by Eduserv.

\section{References}

1. Crossan, A., Brewster, S.: MICOLE - Inclusive Interaction for Data Creation, Visualisation and Collaboration. In: Hands on Haptics Workshop CHI'05 (2005)

2. Jansson, G., Pedersen, P. Obtaining Geographical Information from a Virtual Map with a Haptic Mouse. In: Proceedings of International Cartographic Conference (2005)

3. Kuber, R., Yu, W., McAllister, G.: Towards Developing Assistive Haptic Feedback for Visually Impaired Internet Users. To appear: Proceedings of CHI'07. ACM Press (2007)

4. Kuber, R., Murphy, E., McAllister, G., Strain, P., Yu, W.: Evaluation of an Assistive Web Interface: A Collaborative Approach. Internal Technical Report, (2007)

5. Langdon, P., Hwang, F., Keates, S., Clarkson, P.J., Robinson, P.: Investigating Haptic Assistive Interfaces for Motion Impaired Users: Force Channels and Competitive Attractive Basins. In: Proceedings of Eurohaptics'02, (2002), 122-127

6. Magnusson, C., Danielsson, H., Rassmus-Grohn, K.: Non Visual Haptic Audio Tools for Virtual Environments. In McGookin, D., Brewster, S. (eds.): Haptic and Audio Interaction Design. Lecture Notes in Computer Science, Vol. 4129. Springer-Verlag, (2006), 111-120

7. Murphy, E., Kuber, R., McAllister, G., Strain, P., Yu, W.: An Empirical Investigation into the Difficulties Experienced by Visually Impaired Internet Users. To appear: Universal Access in the Information Society (2007)

8. Mynatt, E., Weber, G.: Nonvisual Presentation of Graphical User Interfaces: Contrasting Two Approaches. In: Proceedings of CHI'94. ACM Press (1994), 166-172

9. RNIB Good Web Design. Available: http://www.rnib.org.uk/xpedio/groups/public/documents/code/public_rnib003460.hcsp

10. Sanchez, J.L., Baloian, N., Hassler, T.: Blind to Sighted Children Interaction through Collaborative Environments. In de Vreede, G.J., Guerro, L.A., Raventos, G.M. (eds.): Groupware: Design, Implementation and Use. Lecture Notes in Computer Science, Vol. 3198, Springer-Verlag (2004) 192-205

11. Sallnäs, E.L., Bjerstedt-Blom, K., Winberg, F., Severinson Eklundh, K.: Navigation and Control in Haptic Applications Shared by Blind and Sighted Users. In McGookin, D., Brewster, S. (eds.): Haptic and Audio Interaction Design. Lecture Notes in Computer Science, Vol. 4129. Springer-Verlag (2006) 68-80

12. Winberg, F.: Supporting Cross-Modal Collaboration: Adding a Social Dimension to Accessibility. In McGookin, D., Brewster, S. (eds.): Haptic and Audio Interaction Design. Lecture Notes in Computer Science, Vol. 4129. Springer-Verlag (2006) 102-110

13. Winberg. F., Bowers, J.: Assembling the Senses: Towards the Design of Cooperative Interfaces for Visually Impaired Users. In: Proceedings of Computer Supported Cooperative Work. ACM Press (2004) 332-341

14. Yu, W., Kuber, R., Murphy, E., Strain, P., McAllister, G.: Novel Multimodal Interface for Improving Visually Impaired People's Web Accessibility. Virtual Reality: 92 (2006) 133-148 